\title{
Task Shifting and Skin Punch for the Histologic Diagnosis of Kaposi's Sarcoma in Sub-Saharan Africa: A Public Health Solution to a Public Health Problem
}

\author{
Miriam O. Laker-Oketta ${ }^{\mathrm{a}, \mathrm{e}}$ Megan Wenger $^{\mathrm{e}}$ Aggrey Semeere $^{\mathrm{a}}$ \\ Barbara Castelnuovo $^{a}$ Andrew Kambugu $^{a}$ Robert Lukande ${ }^{b} \quad$ F. Chite Asirwa ${ }^{d, f}$ \\ Naftali Busakhalad Nathan Buziba ${ }^{d}$ Lameck Diero ${ }^{d}$ Kara Wools-Kaloustian ${ }^{f}$ \\ Robert Matthew Strother ${ }^{g}$ Mwebesa Bwanac Winnie Muyindike ${ }^{c}$ \\ Erin Amerson ${ }^{\mathrm{e}}$ Edward Mbidde ${ }^{\mathrm{a}}$ Toby Maurer $^{\mathrm{e}}$ Jeffrey Martin ${ }^{\mathrm{e}}$ \\ anfectious Diseases Institute, and b Department of Pathology, Makerere University, Kampala, and \\ 'Mbarara University of Science and Technology, Mbarara, Uganda; ${ }^{\mathrm{d}}$ Moi University, Eldoret, Kenya; \\ e University of California, San Francisco, Calif., and ${ }^{\mathrm{f}}$ Indiana University, Indianapolis, Ind., USA; \\ ${ }^{g}$ Canterbury Regional Cancer and Haematology Service, Christchurch, New Zealand
}

\section{Key Words}

Kaposi's sarcoma · HIV/AIDS · Skin punch biopsy · Task shifting · Africa

\footnotetext{
Abstract

Fueled by HIV, sub-Saharan Africa has the highest incidence of Kaposi's sarcoma (KS) in the world. Despite this, KS diagnosis in the region is based mostly on clinical grounds. Where biopsy is available, it has traditionally been excisional and performed by surgeons, resulting in multiple appointments, follow-up visits for suture removal, and substantial costs. We hypothesized that a simpler approach - skin punch biopsy - would make histologic diagnosis more accessible. To address this, we provided training and equipment for skin punch biopsy of suspected KS to three HIV clinics in East Africa. The procedure consisted of local anesthesia followed by a disposable cylindrical punch blade to obtain specimens. Hemostasis is facilitated by Gelfoam ${ }^{\circledR}$. Patients removed the dressing after 4 days. From 2007 to 2013, 2,799 biopsies were
}

performed. Although originally targeted to be used by physicians, biopsies were performed predominantly by nurses $(62 \%)$, followed by physicians (15\%), clinical officers (12\%) and technicians $(11 \%)$. There were no reports of recurrent bleeding or infection. After minimal training and provision of inexpensive equipment (USD 3.06 per biopsy), HIV clinics in East Africa can integrate same-day skin punch biopsy for suspected KS. Task shifting from physician to non-physician greatly increases access. Skin punch biopsy should be part of any HIV clinic's essential procedures. This example of task shifting may also be applicable to the diagnosis of other cancers (e.g., breast) in resource-limited settings.

๑) 2015 S. Karger AG, Basel

\section{Introduction}

Even before the HIV epidemic, sub-Saharan Africa was the epicenter of Kaposi's sarcoma (KS) [1, 2]. The advent of HIV led to an explosion in the incidence of KS

\section{KARGER 125}

(c) 2015 S. Karger AG, Base

0030-2414/15/0891-0060\$39.50/0

E-Mail karger@karger.com

www.karger.com/ocl
Dr. Miriam O. Laker-Oketta

Infectious Diseases Institute

Mulago Hospital Complex, PO Box 22418

Kampala (Uganda)

E-Maildrmiriaml@yahoo.co.uk 
in the region [3], but even today, with increasing availability of therapy for HIV, KS remains common. In the most current data from sub-Saharan Africa, KS is the third most common cancer among men and the fifth most common among women [4]. With an estimated incidence of 260 per 100,000 person-years amongst HIVinfected patients [5] (compare with 150 cases per 100,000 person-years for prostate cancer, the most common cancer in the US), KS qualifies as a public health problem in Africa. Even amongst HIV-infected patients on antiretroviral therapy (ART) in sub-Saharan Africa, the incidence of KS is estimated at 138 per 100,000 person-years [6].

Despite the high frequency of KS and international clinical practice standards dictating that cancers need histologic confirmation, the diagnosis of KS in sub-Saharan Africa has heretofore mainly been based on 'clinical' grounds, i.e. on the macroscopic appearance of lesions on the skin or mucous membranes. For example, in one of the subcontinent's most comprehensive population-based cancer registries (in Malawi), only $17 \%$ of KS diagnoses were histological [7]. The uncommon use of histological diagnosis is likely attributable to the clinician's confidence in macroscopic diagnosis, concerns about bleeding after biopsy, and biopsy traditionally being excisional (meaning that the entire abnormal area is removed). Because the performance of excisional biopsies is limited to surgeons (or in some settings pathologists), who are few in number, their utilization has been limited. This is exacerbated by excisional biopsies requiring equipment sterilization, multiple patient visits, and substantial costs at each step of the process. Clinical diagnosis, while essentially free as part of routine activities, unfortunately has its attendant problems, namely, under- and overdiagnosis. Although the frequency of underdiagnosis of KS due to reliance on clinical diagnosis is unknown, delayed diagnosis is known to lead to significant morbidity and mortality $[8,9]$. On the other hand, as many as $25 \%$ of clinical diagnoses of KS may be false positives $[10,11]$, resulting in potential inappropriate use of chemotherapy (e.g., in one large study from South Africa, 29\% of patients with KS went on to get chemotherapy [9]) as well as the psychological stress of a cancer diagnosis.

Given the difficulties associated with excisional biopsy for KS and diagnosis based solely on clinical grounds, other solutions are needed to increase histologic diagnosis of KS in sub-Saharan Africa. Our objective was to determine whether a simpler approach requiring less specialized personnel, less costly equipment, and less burden to patients - skin punch biopsy - could be adopted by clinicians and thereby improve the utilization of biopsy.

\section{Methods}

\section{Introduction of Skin Punch Biopsy}

In 2007, we introduced skin punch biopsy for KS to the Infectious Diseases Institute (IDI) in Kampala, Uganda, as a clinical service in preparation for recruitment for a clinical trial on the treatment of KS. The IDI, which is a member of the East Africa International Epidemiologic Databases to Evaluate AIDS (IeDEA) Consortium [12], is home to an urban-based, university-affiliated HIV clinic at Uganda's National Referral Hospital. The clinic provides free ART and serves approximately 20,000 HIV-infected adults on-site and another 80,000 through its outreach clinics. The intention was to provide clinicians and patients with same-day, free-of-charge punch biopsies for suspected KS. Equipment for the procedure included the following disposable items: a needle and syringe to administer local anesthesia with $2 \%$ lidocaine (commercially co-mixed with $1: 100,000$ epinephrine to support hemostasis), a 4-mm cylindrical skin punch blade (Acuderm, Fort Lauderdale, Fla., USA), and a suture removal kit (forceps and scissors) to extract the biopsy core and detach it from the underlying dermis. Following biopsy, hemostasis was achieved by manual pressure and Gelfoam ${ }^{\circledR}$, a clot promoter (Pfizer, Kalamazoo, Mich., USA). Suture material was available in the procedure tray in the event that it was required, but suturing was not part of the primary protocol. Patients were instructed to keep the biopsy site clean and dry and remove the dressing after 4 days.

Training for the procedure was originally facilitated by a dermatologist from the US (T.M.) who instructed the physicians at the IDI. In an approximately 1 -hour session, the first 20 min featured didactic coverage of the current epidemiology, clinical presentation, and differential diagnosis of KS. Following this, the biopsy procedure was demonstrated on a pig leg (or non-pig animal if Muslim trainees were present), procured from a local slaughterhouse. Attendees then practiced the procedure on the animal legs. If available during the time of the session, a patient with suspected KS served as a demonstration for the biopsy, which was performed by the trainer. In the week following the training session, the instructor was made available to supervise each practitioner upon his/her initial attempt at biopsy with a patient.

In 2008, based on the initial experience at the IDI, we introduced skin punch biopsy using the same training approach to two other members of the IeDEA Consortium, the Immune Suppression Syndrome (ISS) Clinic in Uganda and the Academic Model Providing Access to Healthcare (AMPATH) network in Kenya. The ISS clinic is a rural-based, university-affiliated HIV clinic at the Mbarara Regional Referral Hospital in southwestern Uganda. It has cared for over 22,000 HIV-infected adults since its inception in 1998. AMPATH provides care to over 150,000 HIV-infected persons in a network of 32 urban and rural clinic sites throughout western Kenya; biopsy training was originally performed at the main oncology clinic at the Moi Teaching and Referral Hospital in Eldoret, Kenya, and later at several other clinics in the network. Based on observations at the IDI that non-physicians were interested in and capable of performing the procedure, clinical officers (equivalent to nurse practitioners or physician assistants in the US) and nurses were included in the training in addition to physicians.

\section{Statistical Analysis}

Descriptive statistics were generated on a variety of biopsy practice characteristics over time (e.g., type of provider who performed the procedure) using Stata version 13 (Stata Corporation, 
Fig. 1. Number of skin punch biopsies performed for the diagnosis of KS at three clinic sites in East Africa from January 2007 to July 2013.

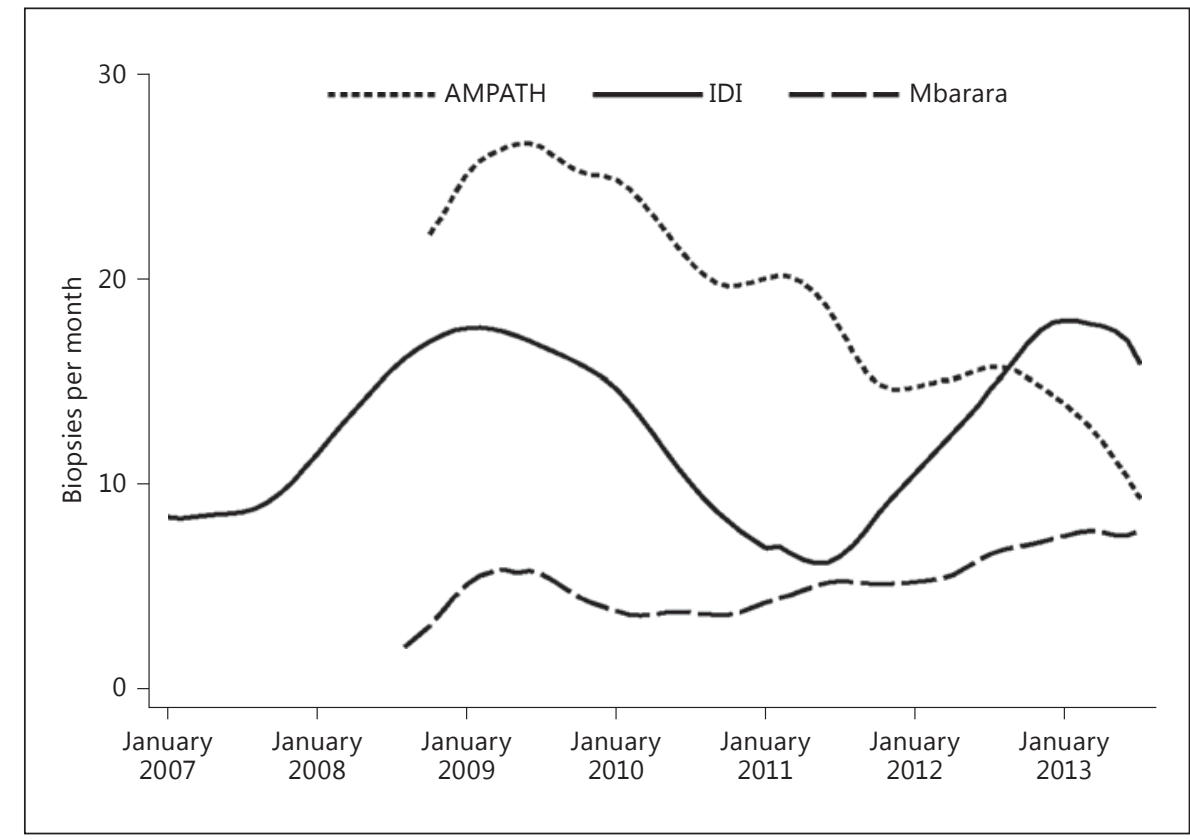

College Station, Tex., USA). Approval to analyze the data collected during the course of clinical care for research was granted by the respective institutional review boards at each of the participating sites.

\section{Results}

From January 2007 to July 2013, a total of 2,799 biopsies were performed across the three sites $(1,360$ at the IDI, 309 at the ISS Clinic Mbarara, and 1,130 at AMPATH). The sites have averaged between about 5 and 25 biopsies per month, although there have been different patterns over time both within and across sites (fig. 1). Overall, $62 \%$ of all biopsies were performed by nurses, $15 \%$ by physicians, $12 \%$ by clinical officers, and $11 \%$ by a laboratory technologist, pharmacy technician or phlebotomist. The proportion of biopsies performed by nonphysicians has increased over time (fig. 2). In 2007, 50\% of all skin punch biopsies were performed by physicians, compared to less than 5\% in 2013 ( $\mathrm{p}<0.001)$.

The procedure has been both uncomplicated to perform and well tolerated by patients. In a consecutive sample of 929 biopsies performed at the IDI in which detailed information on the mode of hemostasis was prospectively recorded, hemostasis was achieved by manual pressure and Gelfoam in $87 \%$ of biopsies, pressure alone in $12.7 \%$, and suture in $0.3 \%$. This sample included several patients with low platelet counts $(1.8 \%$ with $<50,000$ platelets/ $\mu \mathrm{l})$.

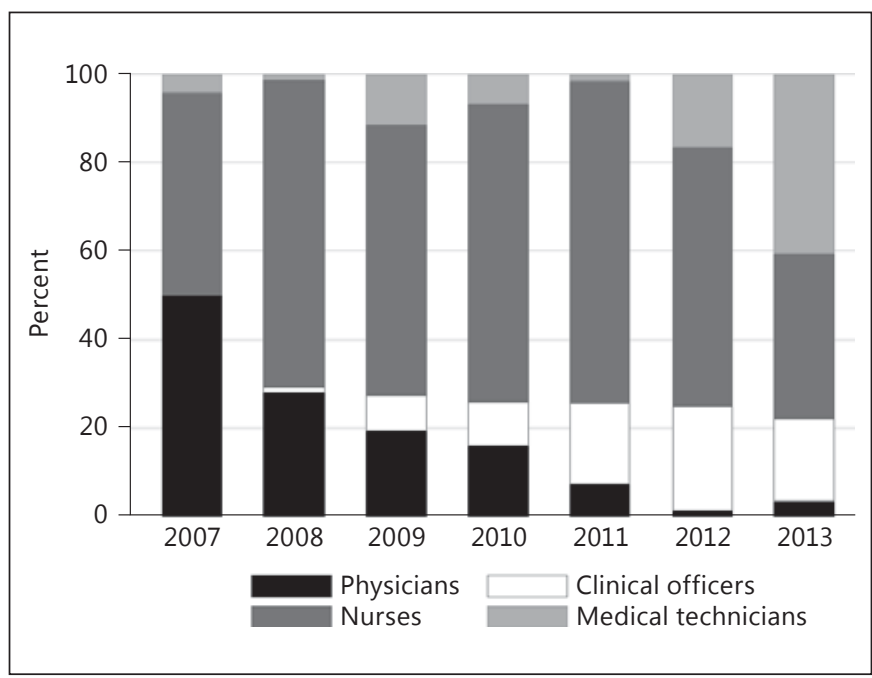

Fig. 2. Number of skin punch biopsies performed according to health care provider position from January 2007 to July 2013 at three clinics in East Africa.

Although limited to a passive recording system, there were no reports of recurrent bleeding after initial hemostasis or subsequent wound infection after the biopsy. The quality of the specimens was good; only $0.4 \%$ of specimens could not be interpreted because of poor quality.

Although training was offered to all primary care clinicians at the three centers with the potential for each to 
perform his/her own procedure, biopsy provision has evolved to be predominantly performed by high-volume service teams at each of the sites. At two of the sites (IDI and ISS Mbarara), the biopsy team has at least one practitioner (a nurse, clinical officer, or medical technician) on call for same-day on-demand procedures, which can typically be performed within $30 \mathrm{~min}$ of referral. At AMPATH, skin punch biopsy is available on a walk-in basis daily at the central oncology clinic in Eldoret, Kenya. At outlying AMPATH primary care sites, several individual clinicians perform biopsies independently, and a member of the biopsy team (a nurse or clinical officer) visits on an established schedule and performs biopsies both on scheduled patients and same-day referrals. Since the initial training by the US dermatologist, local physicians at each of the three sites now provide the training to their colleagues, thus sustaining the expertise. Skin punch biopsy provision has expanded beyond the initial scope at each site. At the IDI, the biopsy service has evolved into a national referral service in which patients from throughout the country can receive free biopsies on a walk-in basis. At ISS Mbarara, biopsy for KS is now a regional referral service for western and southern Uganda, while at AMPATH, biopsies are now available to the entire 2 million person catchment area of western Kenya.

\section{Discussion}

At three large HIV clinics in East Africa, we have shown that skin punch biopsy for suspected KS can be introduced with minimal training, is readily adopted by clinicians, and is safe. Training of local staff only required one visit by one external expert, and the required disposable equipment is inexpensive. Despite initial concerns that clinicians would eschew biopsies because they felt they could accurately diagnose KS macroscopically ('clinically') by visual inspection or that uncontrolled bleeding after biopsy would be common, practitioners were eager to have patients biopsied soon after the introduction at each site. Hemostasis and wound infection have not been problems using this method. Finally, local clinicians have perpetuated training without the need of external assistance.

The most notable evolution we observed in the practice of skin punch biopsy for KS diagnosis was task shifting. When we introduced the procedure, our intention was to train only physicians - the primary practitioners of skin punch biopsy in the US - and we believed that each physician would perform biopsies on his/her own patients as well as the patients cared for by non-physicians (e.g., clin-

Task Shifting and Skin Punch for

Diagnosis of KS in Sub-Saharan Africa ical officers). However, in what was an entirely organic response to physician shortages, each site identified nonphysician providers who, once trained, could safely and effectively perform biopsies. This began with nurses and clinical officers and ultimately spread to technician-level personnel such as phlebotomists. This kind of task shifting has also been demonstrated with cervical cancer diagnosis by non-physicians in sub-Saharan Africa [13] as well as other aspects of HIV care [14]. We hypothesize that these successes in task shifting in KS and cervical cancer diagnosis could translate to other areas of cancer detection that do not require high-level technical skills such as fine needle aspirate of lymph nodes and breast. Task shifting in cancer diagnosis is particularly important in Africa where physicians are limited and overwhelmed with administrative duties [15]. The importance of increasing access to histological diagnosis of cancer in sub-Saharan Africa cannot be overemphasized. Recent data from three cancer registries in sub-Saharan Africa show that only 18$74 \%$ of all cancers are histologically diagnosed [16-18]. Given how clinical diagnoses are often missed by registries, these percentages are likely overestimates.

The inherent simplicity of the skin punch approach coupled with task shifting have combined to make ondemand same-day biopsy for KS a realistic goal for HIV care sites in resource-limited settings. Although we did not formally evaluate the optimal approach to training or organization of biopsy provision, we note that each of the three participating sites chose to organize high-volume service teams comprised of a limited number of practitioners. We suspect that this occurred because any single clinician, limited to his/her own patient pool, may not have the opportunity to perform biopsies often enough to become comfortable with the procedure. Referring all biopsies to a specialized team obviates this. The short-term benefits of having a same-day biopsy are abundant: the skin punch biopsy approach makes formal histologic diagnosis of KS much more accessible and increases the probability of accurate diagnosis. In turn, accurate diagnosis substantiates the need for therapy and can reduce false-positive diagnosis and inappropriate use of chemotherapy for clinical mimickers. We speculate that ready availability of biopsy also holds the promise for earlier detection of KS, which, given the high frequency of advanced disease presentation $[9,19]$, is a major objective in the ultimate control of KS morbidity and mortality. The cost-effectiveness of readily available skin punch biopsy for KS diagnosis has not yet been evaluated, but we suspect it would be favorable considering the high costs associated with $\mathrm{KS}$ when diagnosed in its late stage. 
There are several limitations of our experience. First, financial resources from the US were used to purchase the biopsy equipment. While not expensive (e.g., a punch blade is approx. USD 1.75, and the entire cost of all supplies is USD 3.06 per biopsy), the equipment is not currently part of the stocks of the Ministries of Health in either Uganda or Kenya, and it is unknown whether the public health sector is willing to bear these costs in the future. Second, skin punch biopsy is highly dependent upon available pathology services. Without access to pathologic interpretation, clinicians and patients will not seek biopsies. Indeed, we chose our sites based upon the presence of pathology services, which are unfortunately an exception rather than the rule in East Africa where there is an estimated 1 pathologist for every $1-5$ million people [20,21]. Mitigating this concern, however, is that pathology services need not be directly on site. With the ability of specimens to be preserved in formalin, skin punch biopsy is still useful if the specimen can be sent to a distal laboratory and interpreted quickly. The reality, however, in many parts of sub-Saharan Africa is that even remote pathology services are too far removed, and this is unlikely to change in the near future. Hence, a more immediate solution may rest in point-of-care diagnostics for KS which can assay a skin punch biopsy for KS as an extension of remote pathology services [22]. Third, we did not collect sufficient concurrent information at the clinics to explain the different patterns of numbers of biopsies performed over time at the three sites. Cessation of underlying clinical trials for KS therapy (e.g., at the IDI), backlogs of existing patients with KS needing biopsy soon after the punch procedure was introduced at each site, outreach to surrounding populations outside of the respective clinics, increase of penetrance of ART, and, potentially, unreported supply issues, inconsistent availability of personnel, and changes in clinician interest could have all contributed to the different patterns observed. Fourth, since the initial training in the performance of skin punch biopsy at each of the three sites was performed by a US-trained dermatologist, we do not know whether the uptake at new sites would be as successful when the initial training is performed by a local physician. Fifth, apart from the sample of 929 biopsies, we had no formal mechanism in place to capture complications of the procedure. Finally, while suspected KS can and should be biopsied with the punch technique in order to facilitate an accurate diagnosis, other skin conditions may be contraindicated for biopsy, may need different technical approaches, or may benefit from clinicopathologic direction offered by experienced dermatologists. Hence, our public health approach to KS diagnosis via inexpensive skin punch biopsy equipment and non-physician operators may not be appropriate for other skin diseases.

In conclusion, we have demonstrated that it is feasible to implement skin punch biopsy for the diagnosis of KS in $\mathrm{HIV}$-infected patients in a resource-poor setting. The procedure is well accepted by clinicians, well tolerated by patients, and inexpensive. Importantly, the procedure can be performed safely by non-physicians, which greatly facilitates its utilization. We believe that skin punch biopsy should be part of any HIV clinic's essential procedures, both to accurately diagnose overt suspected KS lesions and to promote early detection of early-stage disease.

\section{Acknowledgements}

We are thankful to Elyne Rotich, Marion Achieng, Christine Ngabirano, the clinic administrators of the IDI, ISS Clinic and AMPATH, and all the clinicians who were involved in the trainings and performed the biopsies. This work was supported by funding from the National Institutes of Health (R01 CA119903, D43 CA153717, P30 AI027763, and U01 AI069911).

\section{Disclosure Statement}

None of the authors have any conflicts of interest to declare.

\section{References}

64
1 Davies JN, Elmes S, Hutt MS, Mtimavalye LA Owor R, Shaper L: Cancer in an African community, 1897-1956. An analysis of the records of Mengo hospital, Kampala, Uganda. Br Med J 1964;1:259-264.

2 Hutt MS, Burkitt D: Geographical distribution of cancer in East Africa: a new clinicopathological approach. Br Med J 1965;2:719722 .
3 Wabinga HR, Parkin DM, Wabwire-Mangen F, Nambooze S: Trends in cancer incidence in Kyadondo County, Uganda, 1960-1997. Br J Cancer 2000;82:1585-1592.

4 Ferlay J, Soerjomataram I, Ervik M, Dikshit R, Eser S, Mathers C, Rebelo M, Parkin DM, Forman D, Bray F: Cancer incidence and mortality worldwide: IARC CancerBase No. 11. Lyon, International Agency for Research on Cancer, 2013. http://globocan.iarc.fr (accessed August 21, 2014) 
5 Semeere A, Wenger M, Busakhala N, Buziba $\mathrm{N}$, Bwana $\mathrm{M}$, Muyindike W, Amerson E, Maurer T, Musick B, Yiannoutsos C, Lukande R, Castelnuovo B, Kambugu A, Glidden D, Vittinghoff E, Wools-Kaloustian K, Martin J: Applying the methods of causal inference to HIV-associated malignancies: estimation of the impact of antiretroviral therapy on Kaposi's sarcoma incidence in East Africa via a nested new user cohort analysis. Presented at the 14th International Conference on Malignancies in AIDS and Other Acquired Immunodeficiencies, November 12-13, 2013, Bethesda, Md., USA.

6 Bohlius J, Valeri F, Maskew M, Prozesky H, Garone D, Sengayi M, Fox MP, Davies MA, Egger M: Kaposi's sarcoma in HIV-infected patients in South Africa: multicohort study in the antiretroviral therapy era. Int J Cancer 2014;135:2644-2652.

7 Banda LT, Parkin DM, Dzamalala CP, Liomba NG: Cancer incidence in Blantyre, Malawi 1994-1998. Trop Med Int Health 2001;6:296304.

-8 El Amari EB, Toutous-Trellu L, GayetAgeron A, Baumann M, Cathomas G, Steffen I, Erb P, Mueller NJ, Furrer H, Cavassini M, Vernazza P, Hirsch HH, Bernasconi E, Hirschel B: Predicting the evolution of Kaposi sarcoma in the highly active antiretroviral therapy era. AIDS 2008;22:1019-1028.

-9 Chu KM, Mahlangeni G, Swannet S, Ford NP, Boulle A, Van Cutsem G: AIDS-associated Kaposi's sarcoma is linked to advanced disease and high mortality in a primary care HIV programme in South Africa. J Int AIDS Soc 2010;13:23-27.
10 Forrestel AK, Naujokas A, Martin JN, Maurer TA, McCalmont TH, Laker-Opwonya MO, Mulyowa G, Busakhala N, Amerson EH: Bacillary angiomatosis masquerading as Kaposi's sarcoma in East Africa. J Int Assoc Provid AIDS Care 2015;14:21-25.

11 Amerson E, Buziba N, Wabinga H, Wenger M, Mwebesa B, Muyindike W, Kyakwera C, Laker M, Mbidde E, Yiannoutsos C, WoolsKaloustian K, Musick B, LeBoit P, McCalmont T, Ruben B, Volberding P, Maurer T, Martin J: Diagnosing Kaposi's sarcoma (KS) in East Africa: how accurate are clinicians and pathologists? Infect Agent Cancer 2012;7(suppl 1):P6.

12 International Epidemiologic Databases to Evaluate AIDS - East Africa. https://www. iedea-ea.org/joomla/ (accessed 2014).

13 Khozaim K, Orang'o E, Christoffersen-Deb A, Itsura P, Oguda J, Muliro H, Ndiema J, Mwangi G, Strother M, Cu-Uvin S, Rosen B, Washington S: Successes and challenges of establishing a cervical cancer screening and treatment program in western Kenya. Int J Gynaecol Obstet 2014;124:12-18.

14 Task shifting. Global recommendations and guidelines. Geneva, WHO Document Production Services, 2008. http://www.who.int/ healthsystems/TTR-TaskShifting.pdf?ua $=1$ (accessed August 20, 2014).

$\checkmark 15$ Benzian H, Cohen LK, Wong TC: Global supply of health professionals. N Engl J Med 2014;370:2246-2247.

16 Jedy-Agba E, Curado MP, Ogunbiyi O, Oga E, Fabowale T, Igbinoba F, Osubor G, Otu T, Kumai H, Koechlin A, Osinubi P, Dakum P, Blattner W, Adebamowo CA: Cancer incidence in Nigeria: a report from populationbased cancer registries. Cancer Epidemiol 2012;36:e271-e278.
17 Laryea DO, Awuah B, Amoako YA, OseiBonsu E, Dogbe J, Larsen-Reindorf R, Ansong D, Yeboah-Awudzi K, Oppong JK, Konney TO, Boadu KO, Nguah SB, Titiloye NA, Frimpong NO, Awittor FK, Martin IK: Cancer incidence in Ghana, 2012: evidence from a population-based cancer registry. BMC Cancer 2014; 14:362.

18 Msyamboza KP, Dzamalala C, Mdokwe C, Kamiza S, Lemerani M, Dzowela T, Kathyola D: Burden of cancer in Malawi; common types, incidence and trends: national population-based cancer registry. BMC Res Notes 2012;5:149.

19 Mwafongo AA, Rosenberg NE, Ng'ambi W, Werner AB, Garneau WM, Gumulira J, Phiri S, Hosseinipour MC: Treatment outcomes of AIDS-associated Kaposi's sarcoma under a routine antiretroviral therapy program in $\mathrm{Li}$ longwe, Malawi: bleomycin/vincristine compared to vincristine monotherapy. PLoS One 2014;9:e91020.

20 Adesina A, Chumba D, Nelson AM, Orem J, Roberts DJ, Wabinga H, Wilson M, Rebbeck TR: Improvement of pathology in sub-Saharan Africa. Lancet Oncol 2013;14:e152-e157.

21 Kingham TP, Alatise OI, Vanderpuye V, Casper C, Abantanga FA, Kamara TB, Olopade OI, Habeebu M, Abdulkareem FB, Denny L: Treatment of cancer in sub-Saharan Africa. Lancet Oncol 2013;14:e158-e167.

22 Mancuso M, Jiang L, Cesarman E, Erickson D: Multiplexed colorimetric detection of Kaposi's sarcoma associated herpesvirus and Bartonella DNA using gold and silver nanoparticles. Nanoscale 2013;5:1678-1686. 